\title{
The NOPTILUS project: Autonomous Multi-AUV Navigation for Exploration of Unknown Environments *
}

\author{
S.Chatzichristofis, A. Kapoutsis, E. B. Kosmatopoulos, * \\ L. Doitsidis, ${ }^{* *}$ D. Rovas, ${ }^{* * *}$ João Borges de Sousa ${ }^{* * *}$ \\ * Department of Electrical and Computer Engineering, Democritus \\ University of Thrace, Xanthi, Greece 83 Informatics \& Telematics \\ Institute, CERTH, Thessaloniki, Greece \\ ** Department of Electronics, Technological Educational Institute of \\ Crete, Chania, Greece 83 Informatics 83 Telematics Institute, CERTH, \\ Thessaloniki, Greece \\ *** Department of Production and Management Engineering, Technical \\ University of Crete, Chania, Greece 83 Informatics 83 Telematics \\ Institute, CERTH, Thessaloniki, Greece \\ **** Faculdade de Engenharia da Universidade do Porto, R. Dr. \\ Roberto Frias, s/n 4200-465 Porto, Portugal
}

\begin{abstract}
Current multi-AUV systems are far from being capable of fully autonomously taking over real-life complex situation-awareness operations. As such operations require advanced reasoning and decision-making abilities, current designs have to heavily rely on human operators. The involvement of humans, however, is by no means a guarantee of performance; humans can easily be over-whelmed by the information overload, fatigue can act detrimentally to their performance, properly coordinating vehicles actions is hard, and continuous operation is all but impossible. Within the European funded project NOPTILUS we take the view that an effective fully-autonomous multi-AUV concept/system, is capable of overcoming these shortcomings, by replacing human-operated operations by a fully autonomous one. In this paper, we present a new approach that is able to efficiently and fully-autonomously navigate a team of AUVs when deployed in exploration of unknown static and dynamic environments towards providing accurate static/dynamic maps of the environment. Additionally to achieving to efficiently and fully-autonomously navigate the AUV team, the proposed approach possesses certain advantages such as its extremely computational simplicity and scalability, and the fact that it can very straightforwardly embed and type of physical or other constraints and limitations (e.g., obstacle avoidance, nonlinear sensor noise models, localization fading environments, etc).
\end{abstract}

Keywords: SLAM-TT, Exploration, AUV, autonomous navigation

\section{INTRODUCTION}

Despite the advances made through current multi-AUV research endeavors, the existing or planned multi-AUV systems are far from being capable of fully autonomously taking over real-life complex situation-awareness operations. Such operations require that the overall system is equipped with reasoning, situation understanding, planning, and decision-making abilities attributes that existing/planned designs are unable to provide. Instead, current designs have to heavily rely on human operators who assign a set of high-level tasks to the AUVs (e.g. a specific set of locations or paths/targets the AUVs have to visit or follow, respectively). As soon as the high-level tasks have been assigned, the existing/planned designs focus on accomplishing them successfully while taking into account

\footnotetext{
* The research leading to these results has received funding from the European Communities Seventh Framework Programme (FP7/20072013) under grant agreement n. 270180 (NOPTILUS).
}

constraints and requirements, such as obstacle-avoidance, energy-consumption, minimum formation error, etc. As such, existing/planned designs do not provide integrated AUV systems that are able to (a) automatically assess and understand the current situation (with regards to the particular mission the AUVs have been deployed for) and (b) autonomously assign tasks/navigate the AUVs so that the overall system accomplishes successfully and fully autonomously the desired mission. Even worse, and mostly due to the involvement of human operators, existing approaches do not provide any guarantees that the overall multi-AUV mission will be accomplished optimally (or, at least, nearly-optimally). Instead, there exist many cases where operator-made decisions are not just far from being optimal but they may even put the success of the overall mission at stake. Within the European funded project NOPTILUS we take the view that an effective fully-autonomous multi-AUV concept/system, is capable of overcoming these shortcomings, by replacing human- 
operated operations by a fully autonomous one. In this paper, we present a new approach that is able to efficiently and fully-autonomously navigate a team of AUVs when deployed in exploration of unknown static and dynamic environments towards providing accurate static/dynamic maps of the environment. Additionally to achieving to efficiently and fully-autonomously navigate the AUV team, the proposed approach possesses certain advantages such as its extremely computational simplicity and scalability, and the fact that it can very straightforwardly embed and type of physical or other constraints and limitations (e.g., obstacle avoidance, nonlinear sensor noise models, localization fading environments, etc). In the heart of the proposed approach lies the so-called Cognitive-based Adaptive Optimization (CAO) algorithm, that has been successfully applied in real-life to the problem of optimal surveillance coverage using swarms of flying robots $(2 ; 9)$.

\section{AUTONOMOUS MULTI-AUV NAVIGATION FOR EXPLORATION OF UKNOWN ENVIRONMENTS}

We consider the problem where $N_{R}$ AUVs are deployed in an underwater environment in order to estimate as accurately as possible the $3 \mathrm{D}$ positions of $N_{L}$ static feature points (landmarks) as well as the (moving) 3D positions of $N_{T}$ dynamic targets. Contrary to aerial or ground robots, the design for exploration using AUVs will have to take into account the very strict limitations of the underwater environment the AUVs operate on: very low bandwidth communications, lack of GPS signals underwater, and very limited visibility of the AUVs vision and sonar sensors are some of the limitations that render multi-AUV autonomous navigation for exploration a very challenging task. Below, we list all different major limitations/challenges that any strategy for such a problem has to take into account:

(LocFade) As underwater there is no GPS signal, the AUVs have to heavily rely on a combination of IMU signals, DVL, etc for localization. Typically, an EKF (or similar) algorithm is employed which is initialized on the surface and - while underwater - it fuses the different signals coming from the on-board AUV sensors (IMU, DVL, etc) as well as from signals coming from the other AUVs. No matter how advanced technologically sensors and communications are employed, the typical situation is that localization is fading, i.e., the error between the actual AUV position and its estimation is diverging. In cases where the error becomes unacceptably large, one or more of the AUVs must somehow re-gain localization e.g., by re-surfacing or by getting in contact with a surface vehicle.

(NL-Noise) The typical assumption made in most robotic applications that the sensor noise is additive Gaussian noise is very restrictive and not realistic in AUV applications: In AUVs sonar- and vision-based sensors, the sensor noise affect the sensor measurements in a NonLinear fashion: typically, the noise affecting such sensors is proportional to the sensor-to-sensing point distance, i.e., the larger is the AUV-to-sensing point distance, the large is the sensor noise. As a result, it is more realistic to assume a multiplicative sensor noise model that takes the form

$$
y=h(x, q)+d(x, q) \xi
$$

where $y$ is the sensor measurement, $x, q$ are the positions of the AUV and the sensing point (landmark/target/another AUV), respectively, $h(x, q)$ is the sensor model in the noisefree case, $d(x, q)$ is the distance between $x$ and $q$ and $\xi$ is a standard Gaussian noise.

(LimVis) In addition to the (NL-Noise) limitation, the AUV vision and sonar sensors are of very limited visibility. As a result, additionally to the nonlinear sensor noise assumption (1), the sensor model for vision and sonar sensors should be augmented to count for the limited visibility constraint. Moreover, the sensor model must be augmented to count for the case where there is no line-ofsight between the AUV and the sensing point (e.g., there is an obstacle in between). As a result, the actual sensor model becomes:

$$
y_{x-q}= \begin{cases}\text { undefined } & \text { if }\|x-q\| \geq \text { thres } \\ \text { undefined } & \text { if there is no line-of- } \\ & \text { sight between } x \text { and } q \\ h(x, q)+d(x, q) \xi & \text { otherwise }\end{cases}
$$

where $y_{x-q}$ denotes the sensor measurement from an AUV at position $x$ to a sensing point at position $q$, thres denotes the visibility threshold beyond which the vision or sonar sensor does not "see" and $\|\cdot\|$ denotes the Euclidean norm.

(ObsAvoid) As in any real-life robot application, the AUV navigation system must make sure that the AUVs avoid obstacles as well as they remain within a prespecified operational area. Usually, it is realistic to assume that the AUVs can detect with accuracy the position of the obstacles nearby; however, obstacle avoidance may have catastrophic consequences to the success of the overall navigation/exploration. See next section for such an example.

(Scalable) Finally, a main issue for any multi-AUV navigation algorithm for exploration is scalability. Of course, scalability is an issue in any multi-robot application. In the case of multi-AUV applications, the scalability issue becomes way more significant mainly due to the limited bandwidth of AUVs communication systems that allow only a few hundreds of bits/second to be transmitted/received.

Having all these limitations in mind, we now proceed to present the proposed methodology along with the remarks on the limitations of existing approaches.

\section{PROBLEM DEFINITION}

Typically, when a single AUV or a team of AUVs is deployed to map an unknown static or dynamic environment, the positions of the landmarks, targets as well as the positions of the AUVs themselves are estimated through a so-called Simultaneous Localization And Mapping and Target Tracking (SLAM-TT) algorithm, which employs an EKF or similar approach to simultaneously estimate all the above-mentioned quantities, see e.g. $(1 ; 7 ; 6)$ and the references there in. Over the past years, very powerful approaches have been developed that can quite efficiently provide the estimates of the landmarks', targets' and AUVs' positions, provided that the trajectories of the AUVs are efficiently designed. However, efficient design of the AUV trajectories is not trivial: in most cases an offline design of the AUV trajectories is performed. Off-line design of the AUV trajectories is, of course, by no means 
a guarantee of performance as the AUVs may enter into highly unobservable states, they may spend "too much time" in areas with no important information for the exploration task, while they may pass very fast through very crucial areas for the exploration task, producing thus a very poor map of these areas, etc.

For this reason, the last few years special attention have been paid in developing techniques for active exploration (also known as active SLAM-TT), see e.g., (11; 10): using the information received so far, the AUV next positions are decided so they optimize the mapping information of the SLAM-TT algorithm. Although, there are many different approaches proposed for doing so, the vast majority of those approaches are based on the following concept: check all feasible next AUV positions [e.g., all next AUV positions that do not violate the (ObsAvoid) constraints as well as constraints that have to do with the maximum allowable AUV speed] and find the ones that optimize some information metric that corresponds to the accuracy of the SLAM-TT algorithm; then, move to the positions that optimize this information metric, and so on. Different types of such information metrics have been proposed, with the most popular being the trace of the EKF error covariance matrix, see e.g., $(11 ; 10)$. In such a case the AUVs are moving to the next positions that minimize the average (expected) EKF estimation error.

There two big issues with all the above mentioned active exploration algorithms: the first is scalability, since it computationally not feasible to check all possible combinations of next AUVs positions. As a matter of fact, such algorithms become practically infeasible even in the single AUV case. There are, of course, many different approaches that relax the computational requirement of checking all possible next positions at the expense of sacrificing efficiency. However, even in the unrealistic case where infinite computing power would be available, as these algorithms are based on EKF - which, in turn, is based on linearizing the nonlinear multi-AUV/environment dynamics - the presence of nonlinear constraints (e.g., for obstacle avoidance or for not leaving a pre-specified area) may be destructive to the efficiency of the overall active exploration mission. The results of such a case are depicted in Figure 1: three AUVs have been deployed for estimating the location of 30 static landmarks and their trajectories are designed so they minimize the trace of the EKF error covariance matrix, while they avoid obstacles (landmarks) and they remain within the cube $[-1,+1]^{3}$. Although, in the time-interval $[0,79]$ the overall algorithm behaves quite efficiently, it starts diverging as soon as the AUVs "hit" the boundaries of the area they have to remain within.

In this paper, we propose a totally different approach to the active exploration problem. In order to describe the proposed approach we need some preliminaries. Let $\mathcal{P}=\left\{x^{(i)}\right\}_{i=1}^{N_{R}}$ denote the configuration of the AUV team, where $x^{(i)}$ denotes the position of the $i$-th AUV. We will say that a landmark or a target $q=(x, y, z)$ is visible if there exists at least one AUV so that

- the AUV and the point $q$ are connected by a line-ofsight;
- the AUV and the point $q$ are at a distance smaller than a given threshold value (defined as the maximum distance the AUVs' sensor can "see").

Given a particular team configuration $\mathcal{P}$, we let $\mathcal{V}$ denote the subset of all visible landmarks and targets, i.e., $\mathcal{V}$ consists of all landmarks and targets $q$ that are visible from the AUVs.

Also, for any landmark or target $q=(x, y, z)$, let $\hat{q}$ denote its estimate as produced by e.g., an EKF. We will say that the landmark or the target $q$ is currently accurately-estimated, if the normed-error $\|q-\hat{q}\| \mid$ is below a certain accuracy threshold. We will denote with $\mathcal{A}$ the set of all landmarks and targets that are currently accurately-estimated. Please note that in case a landmark becomes accurately-estimated then it wil remain accurately-estimated thereafter (i.e., it remains within $\mathcal{A}$ thereafter); however, this is not true for a moving target which may belong to $\mathcal{A}$ at some point and then leave this subset later.

By using the above definitions, we introduce the following $^{1}$ active exploration cost criterion:

$$
\begin{aligned}
J(\mathcal{P})= & \int_{q \in \mathcal{V}, q \notin \mathcal{A}} \min _{i \in\left\{1, \ldots, N_{R}\right\}}\left\|x^{(i)}-q\right\|^{2} d q \\
& +K \int_{q \notin \mathcal{V} \cup \mathcal{A}} d q
\end{aligned}
$$

where $K$ is a user-defined positive constant. Having the AUV team minimizing the above criterion, is equivalent to have the AUVs come as close as possible to those landmarks/targets that are currently visible and have not been accurately-estimated [first term in the RHS of (3)] and, concurrently moving the AUVs so that they "see" those landmarks/targets that are currently not visible and not accurately-estimated [second term in the RHS of (3)]. In other words, the first term is responsible for moving the AUVs closer to the landmarks/targets so that they reduce the sensor noise effect and they can "see them better", while the second term is responsible for moving the AUVs closer to landmarks/targets that "have not seen before" (or "have been poorly seen"). The constant $K$ serves as a weight for giving less or more priority to one of the terms of the RHS of (3).

Please note that if the AUVs' trajectories achieve to render the value of $J$ zero (or sufficiently small), then the overall active exploration mission has been successfully accomplished provided that the position of all AUVs is accurately known. However, as the position of the AUVs [see limitation (LocFade)] is by no means accurately known in AUV missions, the active exploration criterion (3) must

\footnotetext{
1 Please note that the subset $\mathcal{A}$ cannot be calculated in real-life as its calculation requires knowledge of the true landmark/target positions. However, in practice the subset $\mathcal{A}$ can be estimated with high accuracy from e.g., the EKF error covariance matrix (e.g., if all three elements of the diagonal of the EKF error covariance matrix that correspond to a particular landmark/target are below a certain accuracy threshold, then this landmark/target belongs to $\mathcal{A})$. Similarly the term $\int_{q \notin \mathcal{V} \cup \mathcal{A}} d q$ cannot be computed in practice as this term involves those landmarks/targets that are invisible. This problem can be overcome by noticing that $\int_{q \notin \mathcal{V} \cup \mathcal{A}} d q=\int_{q} d q-$ $\int_{q \in \mathcal{V} \cup \mathcal{A}} d q$ and the integral $\int_{q} d q$ is constant.
} 

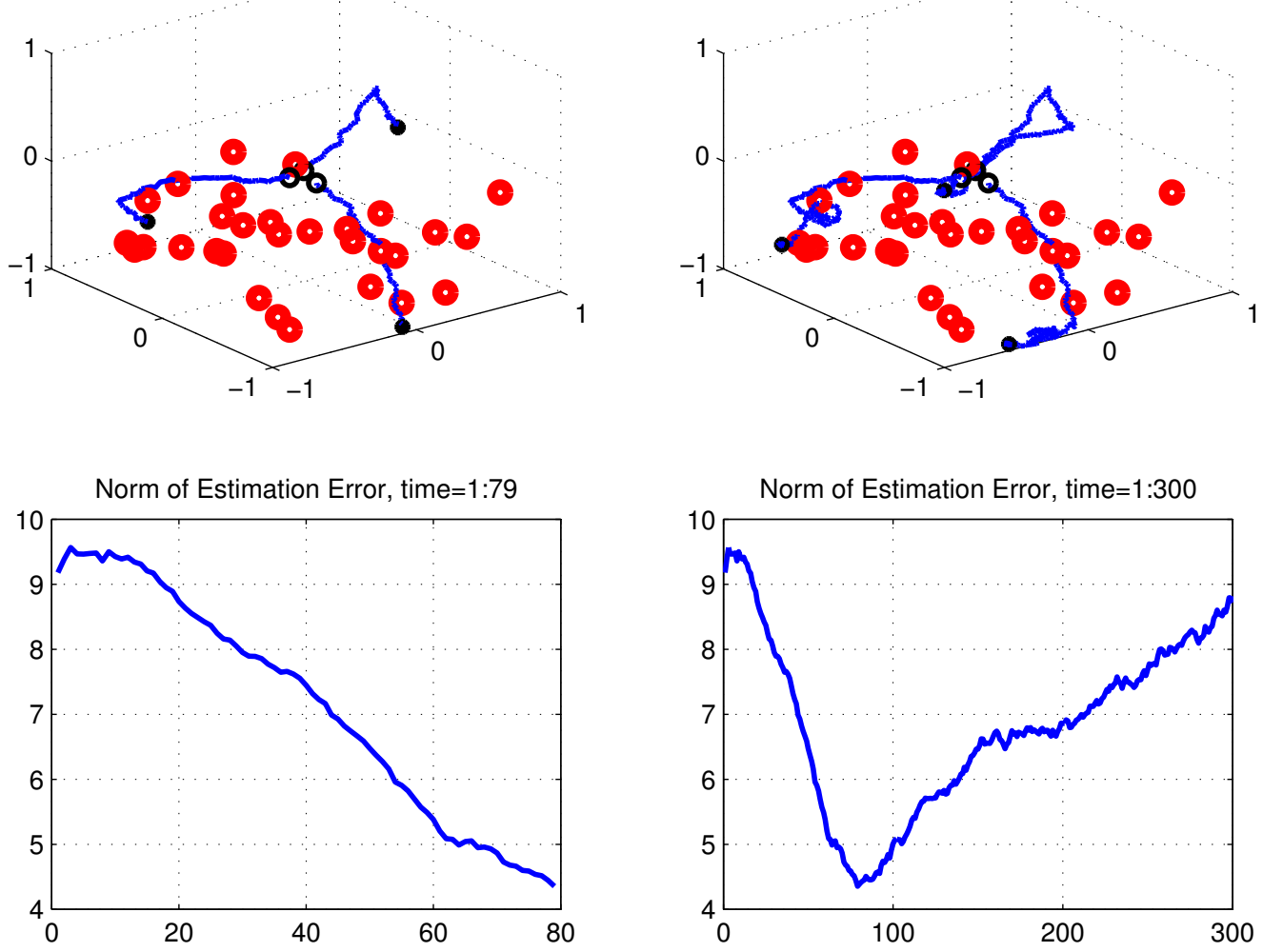

Fig. 1. Autonomous exploration by moving towards minimizing the trace of EKF error covariance matrix: $N_{R}=$ $3, N_{L}=30, N_{T}=0$, (timVis), i.e., by assuming unlimited visibility, perfect localization and infinite computing power. The estimation error starts diverging as soon as the AUVs hit the boundary of the cube $[-1,+1]^{3}$ the AUVs are constrained to remain within. Remark: In figures $\mathbf{1}, \mathbf{2}$ and $\mathbf{5}$ of this paper, the value 1 in the $z$-axis corresponds to the sea-surface and the value -1 corresponds to the maximum allowable AUV depth.

be modified so as to account for this problem. Although there are many different ways to tackle such a problem, one possible way - and this is the one adopted in the simulation experiments to be reported later in this paper - is by assuming the existence of an additional "fictitious" target. The position of this "fictitious" target along the $z$-axis is always on the sea-surface and its $x$ - and $y$-positions are the same with the AUV that is currently closer to the seasurface. Furthermore, it is assumed that this "fictitious" target remains accurately-estimated as long as the AUVs are sufficiently localized: whenever the AUVs' localization accuracy exceeds a certain threshold then the "fictitious" target becomes non-accurately-estimated (but visible) and as a result one of the AUVs is re-surfacing in its attempt to come close to the "fictitious" target [i.e., it attempts to minimize the first term in the RHS of (3)]. A large weight in term of (3) that corresponds to the distance between the "fictitious" target and its closest AUV may be also added in order to make sure that the criterion $J$ gives priority to re-surfacing one of the AUVs in cases of poor localization.

\section{THE COGNITIVE-BASED ADAPTIVE OPTIMIZATION APPROACH}

Having defined the active exploration criterion, we will now proceed on presenting the proposed algorithm for autonomously navigating the AUVs towards minimizing such a criterion. The algorithm to be used is based on the so called Cognitive-based Adaptive Optimization (CAO) approach originated in the referebces $(3 ; 4 ; 5)$, The version of the CAO algorithm used within the proposed approach takes the same form as the one of $(2 ; 9)$ and is a an extension of the original CAO version of presented and analyzed in $(4 ; 5)$. The main difference is that the work of $(2 ; 9)$ extended the CAO approach of $(4 ; 5)$ so that it efficiently takes care of the various constraints of the type (ObsAvoid). Below, we provide the main details of the $\mathrm{CAO}$ algorithm as employed in the framework of the active exploration problem.

We start by noticing that the active exploration criterion (3) is a function of the AUVs positions, i.e.,

$$
J_{k}=\mathcal{J}\left(x_{k}^{(1)}, \ldots, x_{k}^{\left(N_{R}\right)}\right)
$$

where $k=0,1,2, \ldots$ denotes the time-index, $J_{k}$ denotes the value of the active exploration criterion at the $k$-th time-step, $x_{k}^{(1)}, \ldots, x_{k}^{\left(N_{R}\right)}$ denote the position vectors of the AUVs $1, \ldots, N_{R}$, respectively, and $\mathcal{J}$ is a nonlinear function which depends - apart from the AUVs positions - on the particular environment where the AUVs live (e.g., position of landmarks/targets).

Due to the dependence of the function $\mathcal{J}$ on the particular environment characteristics, the explicit form of the function $\mathcal{J}$ is not known in practical situations; as a result, 
standard optimization algorithms (e.g., steepest descent) are not applicable to the problem in hand. However, in most practical cases, like the one treated in this paper, the current value of the active exploration criterion can be estimated from the AUVs sensor measurements. In other words, at each time-step $k$, an estimate of $J_{k}$ is available through AUVs sensor measurements,

$$
J_{k}^{n}=\mathcal{J}\left(x_{k}^{(1)}, \ldots, x_{k}^{\left(N_{R}\right)}\right)+\xi_{k}
$$

where $J_{k}^{n}$ denotes the estimate of $J_{k}$ and $\xi_{k}$ denotes the noise introduced in the estimation of $J_{k}$ due to the presence of noise in the AUVs sensors. Please note that, although it is natural to assume that the noise sequence $\xi_{k}$ is a stochastic zero-mean signal, it is not realistic to assume that it satisfies the typical Additive White Noise Gaussian (AWNG) property even if the AUVs sensor noise is AWNG: as $\mathcal{J}$ is a nonlinear function of the AUVs positions (and thus of the AUVs sensor measurements), the AWNG property is typically lost.

Apart from the problem of dealing with a criterion for which an explicit form is not known but only its noisy measurements are available at each time, efficient AUV navigation algorithms have additionally to deal with the problem of restricting the AUVs positions so that obstacle avoidance constraints are met. In other words, at each time-instant $k$, the vectors $x_{k}^{(i)}, i=1, \ldots, N_{R}$ should satisfy a set of constraints which, in general, can be represented as follows:

$$
\mathcal{C}\left(x_{k}^{(1)}, \ldots, x_{k}^{\left(N_{R}\right)}\right) \leq 0
$$

where $\mathcal{C}$ is a set of nonlinear functions of the AUVs positions. As in the case of $\mathcal{J}$, the function $\mathcal{C}$ depends on the particular environment characteristics (e.g., location of obstacles, terrain morphology) and an explicit form of this function may be not known in many practical situations; however, it is natural to assume that the active exploration algorithm is provided with information whether a particular selection of AUVs positions satisfies or violates the set of constraints (6).

Given the mathematical description presented above, the active exploration problem can be mathematically described as the problem of moving $x_{k}^{(1)}, \ldots, x_{k}^{\left(N_{R}\right)}$ to a set of positions that solves the following constrained optimization problem:

$$
\begin{array}{r}
\text { minimize }(4) \\
\text { subject to }(6) \text {. }
\end{array}
$$

As already noticed, the difficulty in solving, in real-time and in real-life situations, the constrained optimization problem (7) lies in the fact that explicit forms for the functions $\mathcal{J}$ and $\mathcal{C}$ are not available. To circumvent this difficulty, the $\mathrm{CAO}$ approach, appropriately modified to be applicable to the problem in hand, is adopted. Indeed this algorithm is capable of efficiently dealing with optimization problems for which the explicit forms of the objective function and constraints are not known, but noisy measurements/estimates of these functions are available at each time-step. In the following, we describe the CAO approach as applied to the multi-robot coverage problem described above.

As a first step, the CAO approach makes use of function approximators for the estimation of the unknown objective function $\mathcal{J}$ at each time-instant $k$ according to

$$
\hat{J}_{k}\left(x_{k}^{(1)}, \ldots, x_{k}^{\left(N_{R}\right)}\right)=\vartheta_{k}^{\tau} \phi\left(x_{k}^{(1)}, \ldots, x_{k}^{\left(N_{R}\right)}\right) .
$$

Here $\hat{J}_{k}\left(x_{k}^{(1)}, \ldots, x_{k}^{\left(N_{R}\right)}\right)$ denotes the approximation/ estimation of $\mathcal{J}$ generated at the $k$-th time-step, $\phi$ denotes the nonlinear vector of $L$ regressor terms, $\vartheta_{k}$ denotes the vector of parameter estimates calculated at the $k$-th timeinstant and $L$ is a positive user-defined integer denoting the size of the function approximator (8). The vector $\phi$ of regressor terms must be chosen so that it is a univeral approximator, such as polynomial approximators, radial basis functions, kernel-based approximators, etc.

The parameter estimation vector $\vartheta_{k}$ is calculated according to

$$
\vartheta_{k}=\underset{\vartheta}{\operatorname{argmin}} \frac{1}{2} \sum_{\ell=\ell_{k}}^{k-1}\left(J_{\ell}^{n}-\vartheta^{\tau} \phi\left(x_{\ell}^{(1)}, \ldots, x_{\ell}^{\left(N_{R}\right)}\right)\right)^{2}
$$

where $\ell_{k}=\max \left\{0, k-L-T_{h}\right\}$ with $T_{h}$ being a user-defined nonnegative integer. Standard least-squares optimization algorithms can be used for the solution of (9).

As soon as the estimator $\hat{J}_{k}$ is constructed according to (8), (9), the set of new AUVs positions is selected as follows: firstly, a set of $N$ candidate AUVs positions is constructed according to ${ }^{2}$

$$
x_{k}^{i, j}=x_{k}^{(i)}+\alpha_{k} \zeta_{k}^{i, j}, i \in\left\{1, \ldots, N_{R}\right\}, j \in\{1, \ldots, N\},
$$

where $\zeta_{k}^{i, j}$ is a zero-mean, unity-variance random vector with dimension equal to the dimension of $x_{k}^{(i)}$ and $\alpha_{k}$ is a positive real sequence which satisfies the conditions:

$$
\lim _{k \rightarrow \infty} \alpha_{k}=0, \quad \sum_{k=1}^{\infty} \alpha_{k}=\infty, \quad \sum_{k=1}^{\infty} \alpha_{k}^{2}<\infty .
$$

Among all $N$ candidate new positions $x_{k}^{1, j}, \ldots, x_{k}^{N_{R}, j}$, the ones that correspond to non-feasible positions - i.e., the ones that violate the constraints (6) - are neglected and then the new AUVs positions are calculated as follows:

$$
\left[x_{k+1}^{(1)}, \ldots, x_{k+1}^{\left(N_{R}\right)}\right]=\underset{\substack{j \in\{1, \ldots, N\} \\ x_{k}^{i, j} \text { not neglected }}}{\operatorname{argmin}} \hat{J}_{k}\left(x_{k}^{1, j}, \ldots, x_{k}^{N_{R}, j}\right)
$$

The idea behind the above logic is simple: at each timeinstant a set of many candidate new AUVs positions is generated. The candidate, among all feasible ones, that provides the best estimated value $\hat{J}_{k}$ of the coverage criterion is selected as the new set of AUVs positions. The random choice for the candidates is essential and crucial for the efficiency of the algorithm, as such a choice guarantees that $\hat{J}_{k}$ is a reliable and accurate estimate for the unknown function $\mathcal{J}$; see $(4 ; 5)$ for more details. On the other hand, the choice of a slowly decaying sequence $\alpha_{k}$, a typical choice of adaptive gains in stochastic optimization algorithms is essential for filtering out the effects of the noise term $\xi_{k}$ [cf. (5)]. The next theorem summarizes the

\footnotetext{
2 According to $(4 ; 5)$ it suffices to choose $N$ to be any positive integer larger or equal to $2 \times$ [the number of variables being optimized by $\mathrm{CAO}]$. In our case the variables optimized are the robot positions $x_{k}^{(1)}, \ldots, x_{k}^{\left(N_{R}\right)}$ and thus it suffices for $N$ to satisfy $N \geq 2 N_{R} \times$ $\operatorname{dim}\left(x_{k}^{(i)}\right)$.
} 
properties of the CAO algorithm described above; the proof can be found in (9).

Theorem 1. Let $x^{\left(1^{*}\right)}, \ldots, x^{\left(N_{R}^{*}\right)}$ denote any - local - minimum of the constrained optimization problem (7). Let $N \geq 2 N_{R} \times \operatorname{dim}\left(x_{k}^{(i)}\right)$ and, moreover, the vector $\phi$ satisfy the Universal Approximation Property. Assume also that the functions $\mathcal{J}, \mathcal{C}$ are either continuous or discontinuous with a finite number of discontinuities. Then, the CAObased multi-robot coverage algorithm as described above guarantees that the AUVs positions $x_{k}^{(1)}, \ldots, x_{k}^{\left(N_{R}\right)}$ will converge to one of the local minima $x^{\left(1^{*}\right)}, \ldots, x^{\left(N_{R}^{*}\right)}$ almost surely, provided that the size $L$ of the regressor vector $\phi$ is larger than a lower bound $\bar{L}$.

\section{SIMULATION EXPERIMENTS}

In order to test the efficiency of the proposed approach, extensive simulation experiments have been performed. The details of the simulation environment are as follows:

- The number of AUVs, landmarks and targets were chosen according to $N_{R}=3, N_{T}=2, N_{L}=300$ (please note that the actual algorithm assumes an extra "fictitious" target for re-surfacing purposes; see section 3 ). The AUVs are restricted to lie in the cube $[-1,+1]^{3}$; the landmarks are spread randomly in the bottom half of the cube $[-1,+1]^{3}$ and the target trajectories are generated using a zero-acceleration model (11).

- The AUV-to-landmark and AUV-to-target sensors were assumed to be range sensors concatenated by multiplicative noise as follows:

$$
y_{x-q}= \begin{cases}\text { undefined } & \text { if }\|x-q\| \geq \text { thres } \\ \text { undefined } & \text { if there is no line-of- } \\ & \text { sight between } x \text { and } q \\ d(x, q)+d(x, q) \xi & \text { otherwise }\end{cases}
$$

where $\xi$ is a Gaussian noise of variance 0.01 . The visibility thresholds were set equal to 0.4 for the AUVto-landmark sensors and infinite for the AUV-totarget sensors. Also, a line-of-site between the AUV and a landmark/target/another AUV was assumed in case there is no landmark/target or another AUV in a distance less than 0.1 from the line connecting the AUV with the landmark/target/another AUV.

- All AUVs were assumed to have constant orientation which, moreover, does not have any effect on the sensing capabilities or the sensor model (12). Moreover and for simplicity a simple linear model for the AUV dynamics was assumed, and no effect from external disturbances (e.g., currents or turbulences) was considered.

- Finally, a simple model for the GPS-related localization fading was considered. More precisely, it was assumed that the position accuracy of the AUVs decreases proportionally to the total distance traveled by the AUVs; moreover, as soon as one of the AUVs re-surfaces then all of them get perfectly localized. Such an assumption, although over-simplistic, was sufficient in order to test the capabilities of the proposed algorithm to re-surface the AUVs.

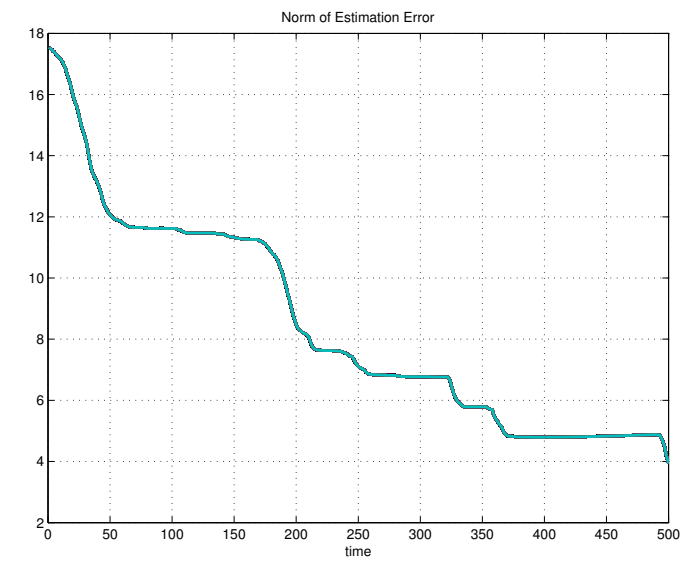

Fig. 3. Autonomous exploration using CAO: $N_{R}=$ $3, N_{L}=30, N_{T}=2$ and by incorporating all of the limitations listed in section 3: Norm of Landmark Estimation Error

Figure 2 exhibits some snapshots of a particular simulation experiment. As it is seen, the CAO-based multi-AUV exploration achieves to estimate accurately most of the landmarks (the black landmarks correspond to the ones that are accurately estimated and the cyan ones to the ones that are non-accurately estimated). Figure 3 exhibits the time-history of the norm of the landmark estimation error for the same experiment. What is really very interesting regarding the behavior of the proposed algorithm can be seen in Figures 4 and 5, respectively: whenever one of the target (resp. the AUVs) becomes non-accuratelyestimated (resp. become poorly localized), the CAO-based algorithm navigates one or more of the AUVs closer to the targets (resp. it resurfaces one of the AUVs) so the target becomes accurately-estimated again (resp. the AUV team becomes accurately localized again).

We close this section, be referring to Figure 6 where the proposed algorithm is compared against the case of a purely random algorithm (i.e., the AUV are randomly choose by making sure that the trajectories do not violate any of the obstacle avoidance, maximum speed, etc, constraints). As a random trajectory motion cannot handle efficiently the cases of target tracking as well as the problem of AUV re-surfacing, in the comparison the AUVs were assumed perfectly localized and there was no target to track (i.e., the AUVs were deployed to perform a pure landmark estimation task). 20 different sets of simulation experiments (random choices for the locations of the landmarks) were executed and Figure 6, clearly exhibits the superiority of the proposed approach.

\section{CONCLUSIONS}

Current multi-AUV systems are far from being capable of fully autonomously taking over real-life complex situationawareness operations. As such operations require advanced reasoning and decision-making abilities, current designs have to heavily rely on human operators. In this paper, we presented a new approach that is capable to efficiently and fully-autonomously navigate a team of AUVs when deployed in exploration of unknown static and dynamic environments towards providing accurate static/dynamic 

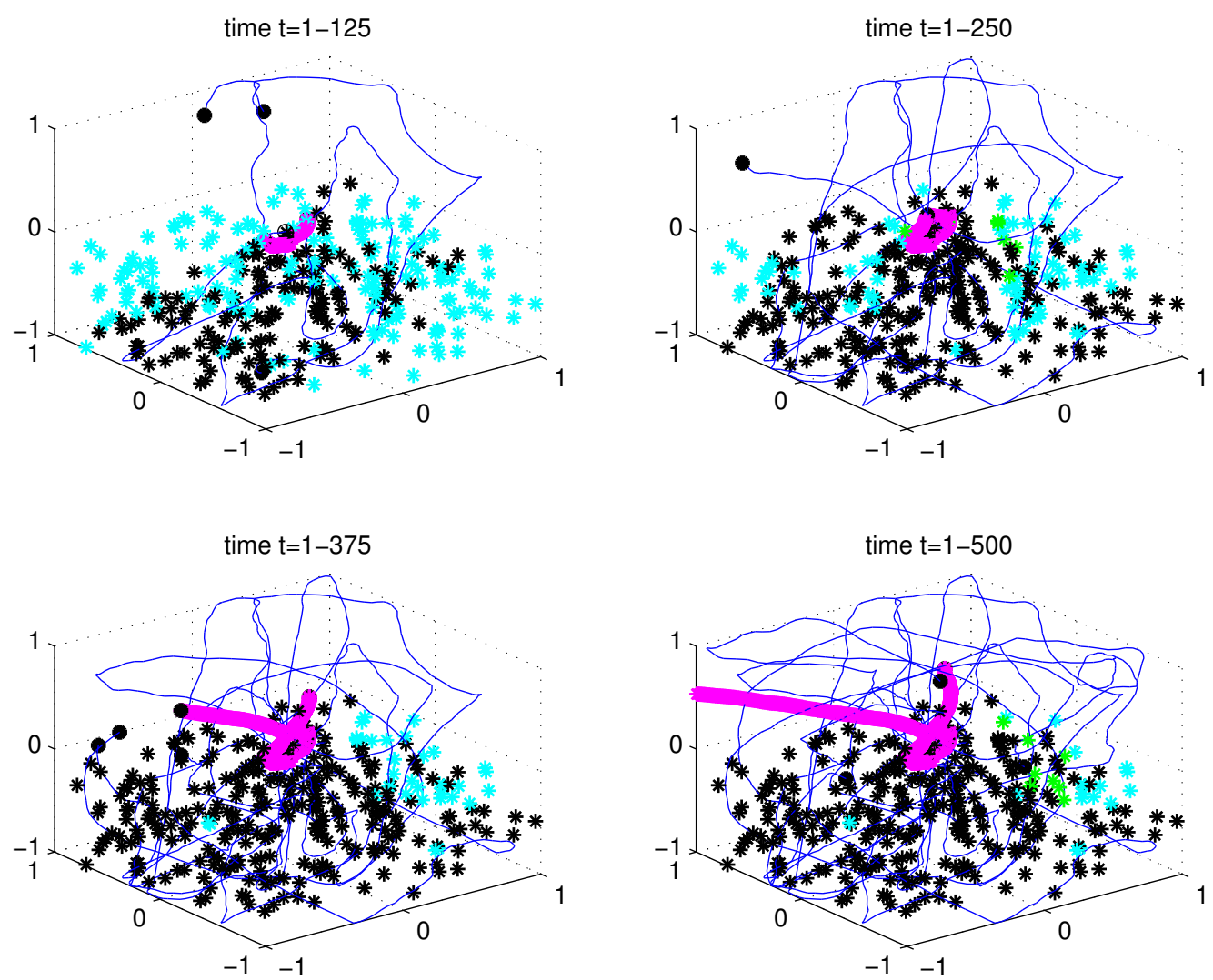

Fig. 2. Autonomous exploration using CAO: $N_{R}=3, N_{L}=30, N_{T}=2$ and by incorporating all of the limitations listed in section 3: AUV trajectories (blue curves), accurately-estimated landmarks (black), non-accurately-estimated landmarks (cyan), non-accurately-estimated landmarks that are currently visible (green) and target trajectories (magenta) at different time-instances of the CAO-based fully-automated multi-AUV exploration.

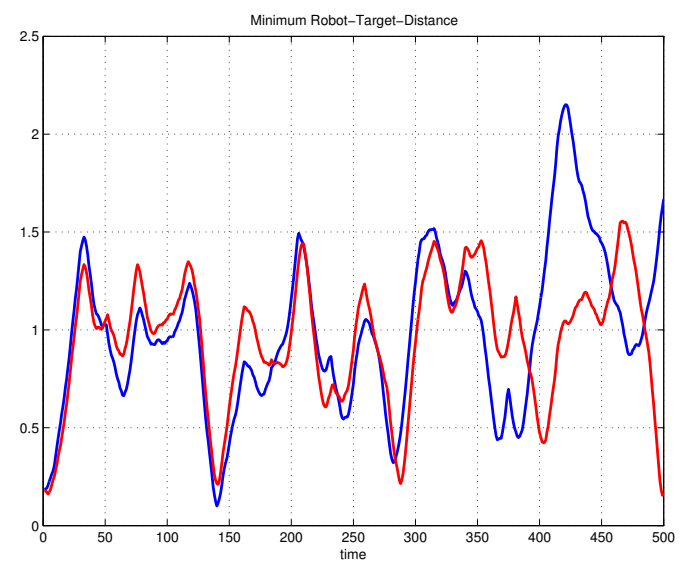

Fig. 4. Autonomous exploration using CAO: $N_{R}=$ $3, N_{L}=30, N_{T}=2$ and by incorporating all of the limitations listed in section 3: Distances between the targets and its closest AUV.

maps of the environment. Realistic simulation experiments exhibited the efficiency of the proposed approach.

\section{REFERENCES}

[1] M. Dissanayake, P. Newman, S. Clark, H. DurrantWhyteand and M. Csobra, "A solution to the simultaneous localization and map building (SLAM) prob-

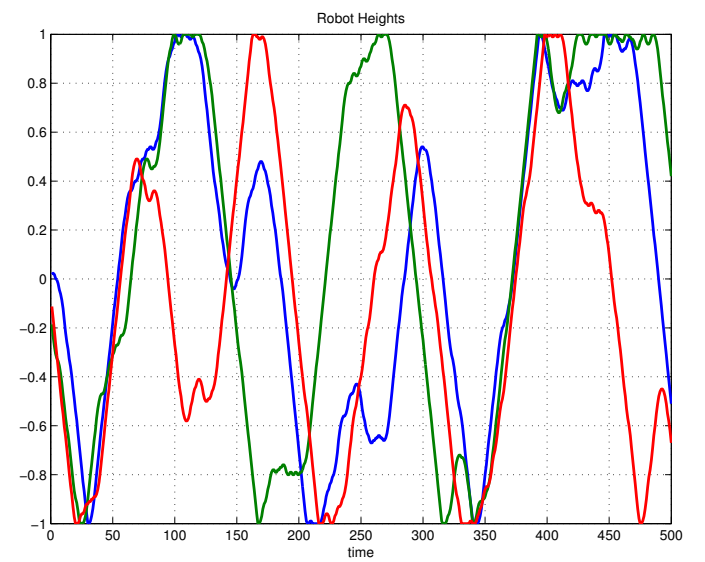

Fig. 5. Autonomous exploration using CAO: $N_{R}=$ $3, N_{L}=30, N_{T}=2$ and by incorporating all of the limitations listed in section 3: Height (depth) of the three AUVs

lem," IEEE Trans. Robotics and Automation, vol. 17, no. 3, pp. 229-241, June 2001.

[2] L. Doitsidis, A. Renzaglia, S. Weiss, E. Kosmatopoulos, D. Scaramuzza, R. Siegwart, "3D Surveillance Coverage Using Maps Extracted by a Monocular SLAM Algorithm," Proceedings of the IEEE International Conference on Robotics and Intelligent System (IROS 2011), 2011. 

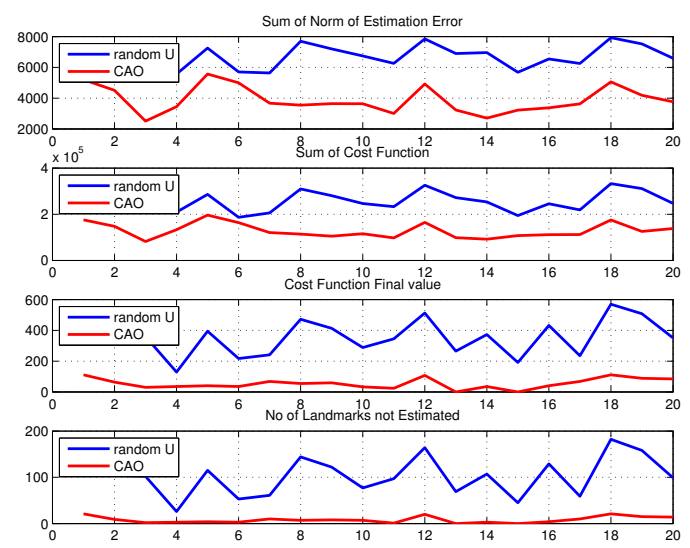

Fig. 6. Autonomous exploration using $\mathrm{CAO}$ and Random trajectories: $N_{R}=3, N_{L}=30, N_{T}=0$ and by incorporating all of the limitations listed in section 3 except : Comparison of different evaluation criteria for 20 different experiments.

[3] E.B. Kosmatopoulos, Papageorgiou, M., Vakouli, A. Kouvelas, A., "Adaptive fine-tuning of nonlinear control systems with application to the urban traffic control strategy TUC," IEEE Transactions on Control Systems Technology, Vol. 15, no. 6, pp. 991-1002, 2007

[4] E.B. Kosmatopoulos, "An adaptive optimization scheme with satisfactory transient performance, $A u$ tomatica, Vol. 45, No. 3, pp. 716-723, 2009.

[5] E.B. Kosmatopoulos and A. Kouvelas, "LargeScale Nonlinear Control System Fine-Tuning through Learning," IEEE Transactions Neural Networks, Vol. 20, No. 6, pp. 1009-1023, 2009.

[6] F.M. Mirzaei and A.I. Mourikisand and S.I. Roumeliotis. "Analysis of Positioning Uncertainty in Cooperate Localization and Target Tracking (CLATT)," August, 2005.

[7] E.D. Nerurkar and S.I. Roumeliotis, "Power-SLAM: A Linear-Complexity, Anytime Algorithm, International Journal of Robotics Research, Special Issue on Stochasticity in Robotics and Biological Systems, 30(6), May 2011, pp. 772-788

[8] A. Renzaglia and L. Doitsidis and A. Martinelli and E.B. Kosmatopoulos, "Cognitive-based Adaptive Control for Cooperative Multi-Robot Coverage", Proceedings of the IEEE International Conference on Robotics and Intelligent System (IROS), 2010.

[9] A. Renzaglia and L. Doitsidis and A. Martinelli and E.B. Kosmatopoulos, "Multi-Robot 3D Coverage of Unknown Areas," The International Journal of Robotics Research, 2012, in press.

[10] R. Sim, and N. Roy, "Global A-Optimal Robot Exploration in SLAM," Proceedings of the IEEE International Conference on Robotics and Automation (ICRA 2005), Barcelona, April 2005.

[11] K. Zhou and S.I. Roumeliotis, "Optimal motion strategies for range-only constrained multi-sensor target tracking," IEEE Transactions on Robotics, vol. 4, no. 5, pp. 1168-1185, Oct. 2008. 